\title{
Automated epileptic seizures detection using multi-features and multilayer perceptron neural network
}

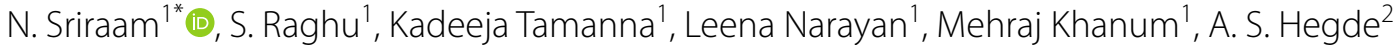 \\ and Anjani Bhushan Kumar²
}

\begin{abstract}
Detection of epileptic seizure activities from long-term multi-channel electroencephalogram (EEG) signals plays a significant role in the timely treatment of the patients with epilepsy. Visual identification of epileptic seizure in long-term EEG is cumbersome and tedious for neurologists, which might also lead to human error. Therefore, an automated tool for accurate detection of seizures in a long-term multi-channel EEG is essential for the clinical diagnosis. This study proposes an algorithm using multi-features and multilayer perceptron neural network (MLPNN) classifier. After appropriate approval from the ethical committee, recordings of EEG data were collected from the Institute of Neurosciences, Ramaiah Memorial College and Hospital, Bengaluru. Initially, preprocessing was performed to remove the power-line noise and motion artifacts. Four features, namely power spectral density (Yule-Walker), entropy (Shannon and Renyi), and Teager energy, were extracted. The Wilcoxon rank-sum test and descriptive analysis ensure the suitability of the proposed features for pattern classification. Single and multi-features were fed to the MLPNN classifier to evaluate the performance of the study. The simulation results showed sensitivity, specificity, and false detection rate of $97.1 \%, 97.8 \%$, and $1 \mathrm{~h}^{-1}$, respectively, using multi-features. Further, the results indicate the proposed study is suitable for real-time seizure recognition from multi-channel EEG recording. The graphical user interface was developed in MATLAB to provide an automated biomarker for normal and epileptic EEG signals.
\end{abstract}

Keywords: EEG, Entropy, Epileptic seizures, MLPNN classifier, Power spectral density, Teager energy

\section{Introduction}

EEG is a clinical procedure carried out for monitoring, diagnosing, and determining neurological disorders related to epilepsy [1]. Epilepsy is a neurological disorder caused due to abnormal electrical discharges in the brain that are characterized by seizures and sudden changes in the electrical activity of the brain. An epileptic seizure is commonly identified as a slow-spike waveform. The unpredicted nature of these seizures makes the daily life immobile with temporary impairments of perception, speech, memory, consciousness and may lead to an increased risk of injury or death [2, 3]. Nearly $4 \%$ of world

\footnotetext{
*Correspondence: sriraam@msrit.edu

${ }^{1}$ Centre for Medical Electronics and Computing, Ramaiah Institute

of Technology (Affiliated to VTU Belgaum), Bengaluru, India

Full list of author information is available at the end of the article
}

population experience seizure at some stage of their life out of which $1 \%$ are epileptic. In interictal recordings, epileptic seizures are usually activated with photostimulation, hyperventilation, and other methods. However, the drawback is that the behavior of provoked epileptic seizures is not necessarily the same as natural ones [4].

The long-term video-EEG recording is a significant milestone to not only capture and analyze ictal events but also help in the contribution of valuable clinical information. Traditional methods of analyzing EEG are time-consuming and a tedious job done by neurologists. Visual interpretation of these long-term EEG recordings can lead to human error and is inefficient [5]. Moreover, the EEG recordings of epileptic seizure are similar to the waves that are a part of background noise and artifacts. For these reasons, automated detection of epileptic 
seizures is needed to reduce the analyzing time and help the neurologists.

The brain is a nonlinear and complex dynamic system, so detecting seizures by a single-channel EEG is not sufficient. Thus, the processing of multi-channel EEG plays a vital role in seizure detection across the brain. However, multi-channel EEG signals impose the challenge of efficiently extracting useful information, and hence, only a few studies have focused on them [6, 7]. An ample number of studies have been proposed for seizure detection. Such technique involves preprocessing, feature extraction, and classification. Selecting significant features is essential to distinguish between normal and epileptic EEG signals. Our focus is on making the job of the neurological experts easy by making the abnormality visually understandable by using the multi-features extraction methods.

Multi-channel EEG recording plays a crucial role in recognizing the epileptic seizure activities from the brain lobes. Automated computed aided screening tool to help neurologist in saving their investigation period and enhance the required clinical diagnosis. Therefore, this study proposes the automated detection of epileptic seizures from multi-channel EEG recordings using multi-features. It also helps neurological experts have a complete picture of the epileptic EEG recordings preventing them from false alarms and leading to decision support with increased accuracy.

Figure 1 shows the flow of the proposed automated seizure detection system. The database was obtained after taking consent from the ethical committee. The raw data that were obtained had other noises such as power-line noise and motion artifacts other than EEG recording. Suitable filtering techniques were implemented to obtain clean EEG. The $50-\mathrm{Hz}$ power-line noise was removed by using a notch filter, a bandpass filter had been

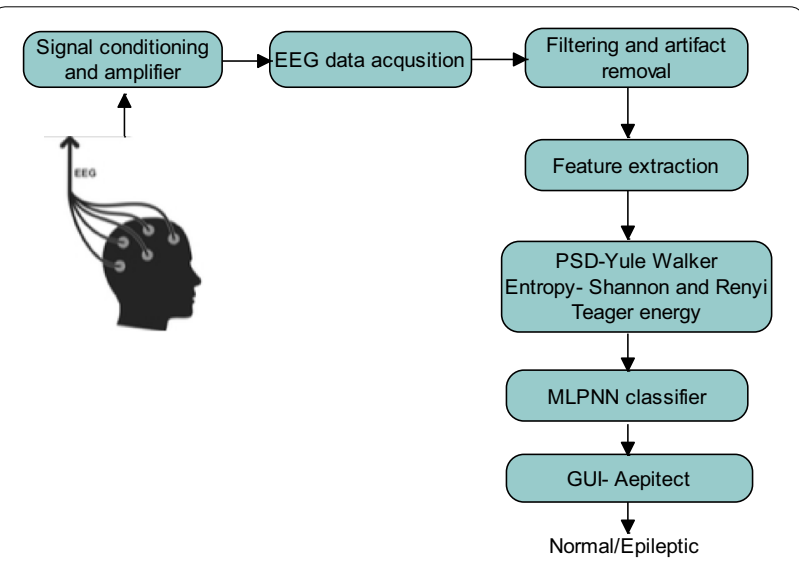

Fig. 1 Block diagram of the proposed Aepitect technique implemented to get the signals in the range of $0.5-40 \mathrm{~Hz}$, and independent component analysis (ICA) was applied to remove the motion artifacts.

The EEG data consisting of both normal and epileptic data annotated by the clinician were segmented separately for offline analysis. The features of interest to evaluate the epileptic EEG, namely PSD, entropy, and TE, were extracted, and descriptive analysis was carried out. The extracted features were given as input to the MLPNN binary classifier. Finally, a graphical user interface (GUI) has been developed to label the signals as normal or epileptic.

So far, several automated epileptic seizure detection methods have been proposed. In the early 1980s, the automated seizure detection procedure for a long duration of EEG recordings was initiated [8]. Guo et al. [9] proposed a line length of EEG as a feature and artificial neural networks classifier-based automated detection of epileptic seizure. The database considered was subjected to preprocessing, visual inspection, and artifact removal. EEG was decomposed into different sub-bands using discrete wavelet transform (DWT), and line length feature was extracted. The classification was done using a threelayer MLPNN, and a classification rate of more than $95 \%$ was achieved. Back-propagation neural network classifier with periodogram and autoregressive features was proposed [10]. Orhan et al. [11] used DWT-based features with MLPNN model for automated detection of epileptic seizures. Kamath [2,3] proposed Teager energy as a quantitative feature for EEG signals. The study used the University of Bonn database to extract Teager energy and compared the classification outcome with Higuchi's fractal dimension and sample entropy. It has been proved that TE provided an accuracy rate of $97.8 \%$, and it can be used in real-time automated applications.

Gurwinder et al. [12] proposed a study to detect epileptic seizures using wavelet transformation and spikebased features. The work used University of Bonn database and wavelet transformation as its preprocessing technique. Spike-based parameters were extracted from both normal and interictal data. MLPNN was used for classification which gave an accuracy of $98.6 \%$. Epileptic seizure detection method was developed using autoregressive modeling [13] and that showed the classification accuracy of $84.2 \%$ using MLPNN. Hierarchical EEG classification system using best basis-based wavelet packet entropy method was proposed [14]. Abbasi and Esmaeilpour [4] proposed a study to choose statistical characteristics of brain signals for detection of epileptic seizures using DWT and perceptron neural network. Their study used University of Bonn database and DWT as a feature extraction method. Statistical characteristics are derived, and a multi-perceptron 
neural network was used as a classifier which gave an accuracy of $98.33 \%$.

The features such as mean, standard deviation, skewness, kurtosis, and the median in the first and second derivative of EEG signals were extracted for mobilebased automated epileptic seizure detection using k-means clustering technique [15]. Bogaarts et al. [16] extracted features such as curve length, root mean square, band power, zero crossing, Hjorth parameters, and Teager energy to classify epileptic EEG from normal using the support vector machine (SVM) classifier. Empirical mode decomposition (EMD) followed by DWT was applied on EEG signals to compute log energy entropy. The obtained features were classified using K-NN classifier, which yields the accuracy of 89.4\% [17]. In the recent study [18], significant features were selected from neighborhood component analysis for the classification of focal and non-focal EEG signals. The highest classification accuracy of $96.1 \%$ was obtained using SVM classifier.

Other studies have introduced approximation entropy [19], $\log$ and norm entropy [20, 21], variants of entropies [22, 23], DWT-based features [24], time-frequencydomain features [25], Shannon, Renyi, log entropy and spectral entropy [20,21], spectral and statistical features [26], and the classification of epileptic seizures. An optimal configuration of MLPNN was derived for the classification of epileptic seizures [27].

It was inferred from various studies that automated seizure detection was based on using single feature extraction. However, using multi-features would help in better classification of normal and epileptic data and classification accuracy.

\section{Materials and methods}

\subsection{EEG data acquisition}

The EEG recordings used in this study were obtained from Ramaiah Memorial College and Hospital, Bengaluru, after getting consent from the ethical committee. Unipolar multi-channel (19 channels) EEG recordings from 20 patients (11 male and nine female), each of 20-min duration, were considered for the study. International 10-20 system was used for the electrode placement, and data were recorded at a sampling rate of $128 \mathrm{~Hz}$. The data, consisting of both normal and epileptic seizures annotated by clinician, were segmented separately for offline analysis. The 19 channels include the recordings from the following placement of the electrodes: Fp1, Fp2, F7, F3, Fz, F4, F8, T3, C3, Cz, C4, T4, $\mathrm{P} 3, \mathrm{Pz}, \mathrm{T} 6, \mathrm{O} 1$, and O2. Table 1 shows each patient information used in our study.
Table 1 Information of each patient EEG data used in our work

\begin{tabular}{|c|c|c|c|}
\hline Patient no. & Sex & Age & Seizure type \\
\hline 1 & $M$ & 80 & SPS, CPS, GTCS \\
\hline 2 & $\mathrm{~F}$ & 20 & SPS, CPS, MCS \\
\hline 3 & $\mathrm{~F}$ & 5 & SPS, CPS \\
\hline 4 & $M$ & 11 & SPS, CPS \\
\hline 5 & $\mathrm{~F}$ & 12 & CPS, GTCS \\
\hline 6 & $\mathrm{~F}$ & 26 & SPS, CPS, MCS \\
\hline 7 & $\mathrm{~F}$ & 7 & SPS, CPS, GTCS \\
\hline 8 & $M$ & 3 & SPS, CPS \\
\hline 9 & $M$ & 12 & SPS, CPS, MCS \\
\hline 10 & $M$ & 44 & SPS, CPS, GTCS \\
\hline 11 & $M$ & 13 & SPS, CPS, MCS \\
\hline 12 & $M$ & 16 & CPS, MCS \\
\hline 13 & $M$ & 5 & CPS, MCS, GTCS \\
\hline 14 & $\mathrm{~F}$ & 7 & SPS, CPS, GTCS \\
\hline 15 & $\mathrm{~F}$ & 9 & CPS, GTCS \\
\hline 16 & $M$ & 6 & SPS, CPS \\
\hline 17 & $M$ & 5 & SPS, CPS, GTCS \\
\hline 18 & $\mathrm{~F}$ & 13 & SPS, CPS \\
\hline 19 & $\mathrm{~F}$ & 21 & SPS, CPS, MCS \\
\hline 20 & M & 18 & CPS, MCS \\
\hline
\end{tabular}

M Male, F Female, SPS Simple partial seizure, CPS Complex partial seizure, GTCS Generalized tonic-clonic seizure, MCS Myoclonic seizure

\subsection{Preprocessing}

Suitable filtering techniques were introduced to eliminate noise and artifacts. An infinite impulse response (IIR) notch filter of order 2 was implemented to remove the $50-\mathrm{Hz}$ power-line noise. A bandpass filter of order 5 with a higher cutoff frequency of $40 \mathrm{~Hz}$ and a lower cutoff frequency of $0.5 \mathrm{~Hz}$ was implemented to retain the EEG rhythms of interest in the data. The filter design specifications are: the passband ripple and attenuation in the stop band were set to $3 \mathrm{~dB}$ and $40 \mathrm{~dB}$, respectively. Artifacts were removed from the filtered EEG using joint approximation diagonalization of eigenmatrices-based ICA technique [28-30].

\subsection{Feature extraction}

Selecting significant features is essential for the proper classification of epileptic seizures. The number of extracted features should be less and easy to compute with reduced computational time. The significant characteristic of an epileptic EEG is a slow wave followed by a spike. The epileptic EEG varies significantly from that of a normal EEG in frequency, period, complexity, etc. Considering all these parameters, the following features were selected for our research work: power spectral density, entropy (Shannon and Renyi entropy), and Teager energy [19-23]. 
In this paper, PSD was used as the power of the EEG signal increased during epileptic activity. Entropy is a measure of the complexity or uncertainty of a signal, higher during epileptic activity, and gives a clear distinction between normal and epileptic. Teager energy depends on the amplitude of the epileptic data that is higher than that of the normal signal. From the preliminary study, it was identified that PSD using Yule-Walker method showed better results as compared to the other methods of PSD like Welch method, Burg's method, and Thomson's method.

\subsubsection{Yule-Walker method}

Yule-Walker method is an autoregressive (AR) method that estimates spectra with narrow peaks by placing the poles of the polynomial close to unity. Narrowly banded spectra are quite common in practice. Hence, this has been chosen as the best method for feature extraction for the study. The AR parameters are represented as $\theta$ by forming a biased estimate of the signal's autocorrelation function and a minimization of a prediction error [31].

For this study, a fourth-order autoregressive model was used to produce the PSD estimates. The preprocessed signal was segmented at a length of $0.5 \mathrm{~s}$, followed by obtaining the PSD estimates, and then, the maximum PSD of each segment was determined. This process was carried out in the complete study.

\subsubsection{Shannon entropy}

It is a measure of the randomness or disorder in physical systems or the amount of average information gained by observations of disordered systems. It is the best possible lossless compression and gives low entropy values for varied distribution and high entropy values when outcomes are uniformly distributed. Shannon's entropy is given by the equation [32]:

$$
E=-\sum p_{i} \log _{2} p_{i}
$$

where $p i$ is the probability of occurrence of the signal.

\subsubsection{Renyi entropy}

It is a generalized form of Shannon's entropy when the Renyi estimation factor $\alpha=1$. It is also called quadratic entropy as $\alpha=2$. The value of $\alpha$ is estimated to be taken as 2 as peak accuracy is achieved with specificity higher than for Shannon's case. Renyi's entropy equation is given as [33]:

$$
E=\frac{1}{1-a} \sum \log _{2} p_{i}^{a}
$$

where $\alpha \geq 0$ and $\alpha \neq 1$.

There are a few special cases in case of Renyi entropy. They include:

$$
\begin{aligned}
& \alpha=0 \text {-obtains maximum entropy. } \\
& \alpha=1 \text {-recovers the Shannon's entropy. } \\
& \alpha=\infty \text {-obtains the minimum entropy. }
\end{aligned}
$$

It can also be added that when $\alpha$ has larger positive value, it is sensitive to events that occur often and when $\alpha$ has larger negative value, and it is sensitive to events that occur seldom.

Since entropy is a function of probability, in this study, the probability was estimated using the histogram method by setting the bins with a uniform width.

\subsubsection{Teager energy}

Teager energy is a nonlinear operator, which can be used for energy estimation of a non-stationary signal. This feature is extremely sensitive to amplitude and frequency changes of a signal. The method is computationally very efficient, as it requires only three samples at any given instance to calculate the physical energy. Since the EEG signal is non-stationary, Teager energy operator can be used as a discriminating feature for normal and epileptic data set.

As per the Teager algorithm, the Teager energy (TE) is estimated from the signal $x(n)$ through the formation of time-delayed state-spaced vectors $x(n)=\left[x_{1}, x_{2}, x_{3}, \ldots\right.$, $\left.x_{n-1}, x_{n}\right]$ where $\mathrm{n}$ is the data points as follows [34]:

$$
\mathrm{TE}=\frac{1}{N-1} \sum_{n=2}^{N-1} x_{n}^{2}-x_{n-1} * x_{n+1}
$$

where $N$ is taken to be 64 (segmentation length of $0.5 \mathrm{~s}$ ).

From the equation, it is clear that Teager energy takes into account the amplitude and the corresponding frequency to determine the physical energy.

\subsection{Descriptive analysis}

Descriptive analysis was performed on the extracted feature samples obtained from epileptic and normal data. The mean, standard deviation (SD), minimum, maximum, interquartile range (IQR), first quartile (Q1), median (Q2), third quartile (Q3), and semi-interquartile deviation (SID) were estimated for extracted features using box plot. The $p$ and $z$ values were found for individual patients for normal and epileptic feature values. The $p$ value should be less than 0.05 which gives a confidence level of greater than $95 \%$, and the $z$ value should be less 
than 1.96 and greater than -1.96 [34]. The descriptive analysis of extracted features showed that the obtained features are significant for further analysis.

\subsection{Classifier}

MLPNN is a feed-forward neural network, which was used for binary classification of the EEG signal. It contains three consecutive layers, namely input, hidden, and output layer [35-38]. In this study, we have used the MLPNN model with a single hidden layer of 10 neurons. Hyperbolic tangent and tangent sigmoid were used as input to hidden and hidden to output activation function, respectively. A scaled conjugate gradient back-propagation was used as a training function. The classification target was set to 0 for normal and 1 for epileptic [27].

\subsection{Performance evaluation}

The performance of the proposed method was evaluated based on the sensitivity $\left(S^{+}\right)$, specificity $\left(S^{-}\right)$, and false detection rate (FDR) for individual patients as follows $[11,19,25]$ :

$$
S^{+}=\frac{\text { Correctly detected epileptic seizures }}{\text { Total number of epileptic seizures }}
$$

$$
S^{-}=\frac{\text { Correctly detected normal activities }}{\text { Total number of activities }}
$$

$$
\text { FDR (per hour })=\frac{\text { Number of false detections }}{\text { Total length of the data }}
$$

\section{Results}

This study takes into account 20 patients' multi-channel EEG recordings. Notch filter and bandpass filters with appropriate cutoff frequencies were used to remove line noise of $50 \mathrm{~Hz}$ and other background noises. ICA was used to remove motion artifacts, and artifact-removed multi-channel EEG is shown in Fig. 2. To maintain the uniformity of the signal, the EEG was segmented at $0.5 \mathrm{~s}$ duration.

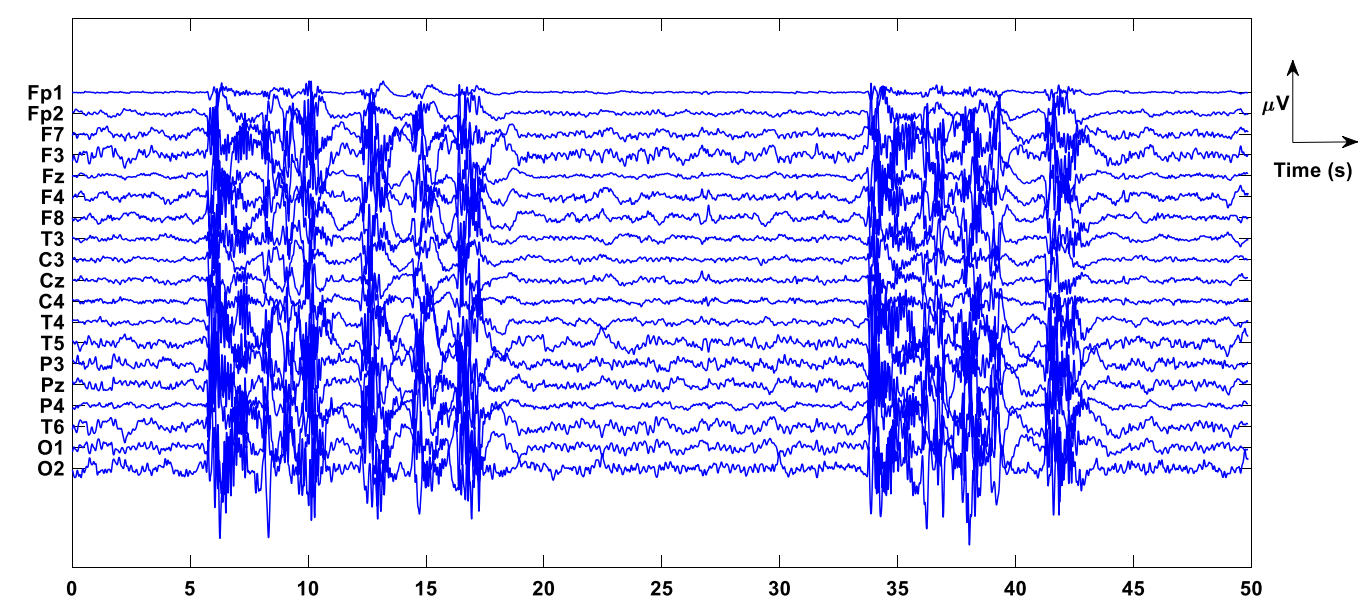

Fig. 2 Preprocessed multi-channel EEG signal

Table 2 Descriptive analysis of extracted features

\begin{tabular}{llcclllllrr}
\hline Feature & EEG & Mean & STD & Min & Q1 & Q2 & Q3 & IQR & Max & SID \\
\hline Yule-Walker PSD & $\mathrm{N}$ & 0.6027 & 1.0179 & 0.0018 & 0.1536 & 0.3402 & 0.5719 & 0.4183 & 15.063 & 0.2091 \\
& $\mathrm{E}$ & 20.866 & 19.7967 & 0.1609 & 7.0496 & 15.703 & 27.513 & 20.4636 & 259.5852 & 10.2318 \\
Shannon entropy & $\mathrm{N}$ & 3.2285 & 0.5698 & 1 & 2.9579 & 3.4082 & 3.6565 & 0.6986 & 4.1804 & 0.3493 \\
& $\mathrm{E}$ & 2.0793 & 0.5666 & 0.9016 & 1.6943 & 2.0441 & 2.4196 & 0.7252 & 3.8029 & 0.3626 \\
Renyi entropy & $\mathrm{N}$ & 3.4005 & 0.7148 & 0.8493 & 3.1329 & 3.4929 & 3.8295 & 0.6966 & 5.3897 & 0.3483 \\
& $\mathrm{E}$ & 5.8863 & 0.5731 & 2.9858 & 5.5831 & 6.1188 & 6.2974 & 0.7142 & 6.7119 & 0.3571 \\
Teager energy & $\mathrm{N}$ & 0.7101 & 2.9253 & 0.0012 & 0.0719 & 0.1917 & 0.4419 & 0.3700 & 80.621 & 0.1850 \\
& $\mathrm{E}$ & 19.24708 & 51.654 & 0.0337 & 3.6780 & 7.0098 & 13.378 & 9.7003 & 720.1391 & 4.8501 \\
\hline
\end{tabular}

' $\mathrm{N}$ ' stands for normal and ' $\mathrm{E}$ ' stands for a person with epilepsy 
Table 3 Wilcoxon rank-sum test results

\begin{tabular}{lcc}
\hline Feature name & $\boldsymbol{p}$ value & $\boldsymbol{z}$-score \\
\hline Yule-Walker PSD & $<0.05$ & -50.8174 \\
Shannon entropy & $<0.05$ & 49.87157 \\
Renyi entropy & $<0.05$ & -51.2641 \\
Teager energy & $<0.05$ & -51.2896 \\
\hline
\end{tabular}

Table 4 Epileptic seizure detection results using the proposed method

\begin{tabular}{llll}
\hline Feature name & $\boldsymbol{S}^{+}$ & $\boldsymbol{S}^{-}$ & FDR $\left(\mathbf{h}^{\mathbf{- 1}}\right)$ \\
\hline Yule-Walker PSD & 86.5 & 94.4 & 3 \\
Shannon entropy & 86.9 & 80.0 & 3 \\
Renyi entropy & 96.2 & 95.2 & 2 \\
Teager energy & 82.0 & 94.8 & 3 \\
Multi-features & 97.8 & 96.4 & 1 \\
\hline
\end{tabular}

A descriptive analysis of the obtained feature from epileptic and normal data was performed. Table 2 shows that the statistical parameters for both epileptic and normal EEG samples obtained from patients were highly distinguishable. Results show that PSD, entropy, and Teager energy in epileptic EEG were more compared to that of normal EEG. A $p$ value was found between normal and epileptic extracted feature samples using a two-sided Wilcoxon rank-sum test. For all the features, $p$ value was found to be less than 0.05 and $z$ value greater or lesser than the prescribed limits, which indicates that all the features were suitable for classification. Table 2 shows the obtained $p$ and $z$ values (Table 3 ).
The classifier was trained using holdout cross-validation method with the ratio of $70-30$ used for training and testing. Highest sensitivity and specificity of $86.2 \%$ and $95.2 \%$ were obtained using Renyi entropy for individual features, respectively. Further, sensitivity, specificity, and FDR of $97.8 \%, 96.4 \%$, and $0.15 \mathrm{~h}^{-1}$ were recorded using multi-features which were highest than all other combinations. Table 4 shows the classification results of the proposed system for all individual features and multifeature combination.

Figure 3 shows the ROC curve obtained from the classification results of PSD, Shannon entropy, Renyi entropy, Teager energy, and multi-features. Maximum AUC of 0.97 was obtained for multi-features, whereas a minimum of 0.83 attained for Shannon entropy. Classification results revealed that the highest performance measures were achieved using multi-features than the single features with the betterment of sensitivity, specificity, and FDR.

\section{Discussion}

The foremost objective of this study was to introduce an automated detection of epileptic seizures using multichannel EEG. Four features, namely PSD, variants of entropy, and Teager energy, were utilized followed by MLPNN classifier. These features were selected for the study based on previous performance on other databases. Experimental results show that multi-features perform better as compared to single features. Figure 4 shows the best validation performance of MLPNN classifiers for multi-features. It can be seen that the best validation performance of 0.08 was obtained at epoch 54. Further, Fig. 5 shows the error histogram of training, validation,

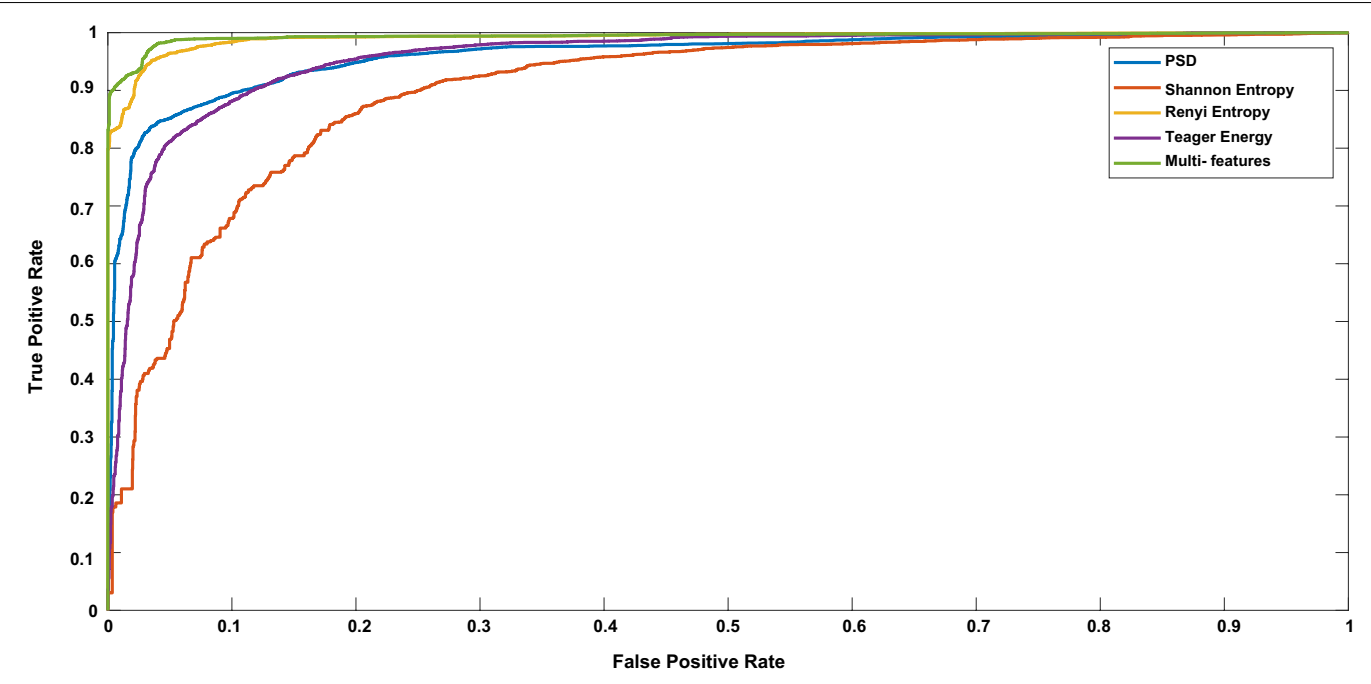

Fig. 3 ROC curve for different features obtained from EEG data 


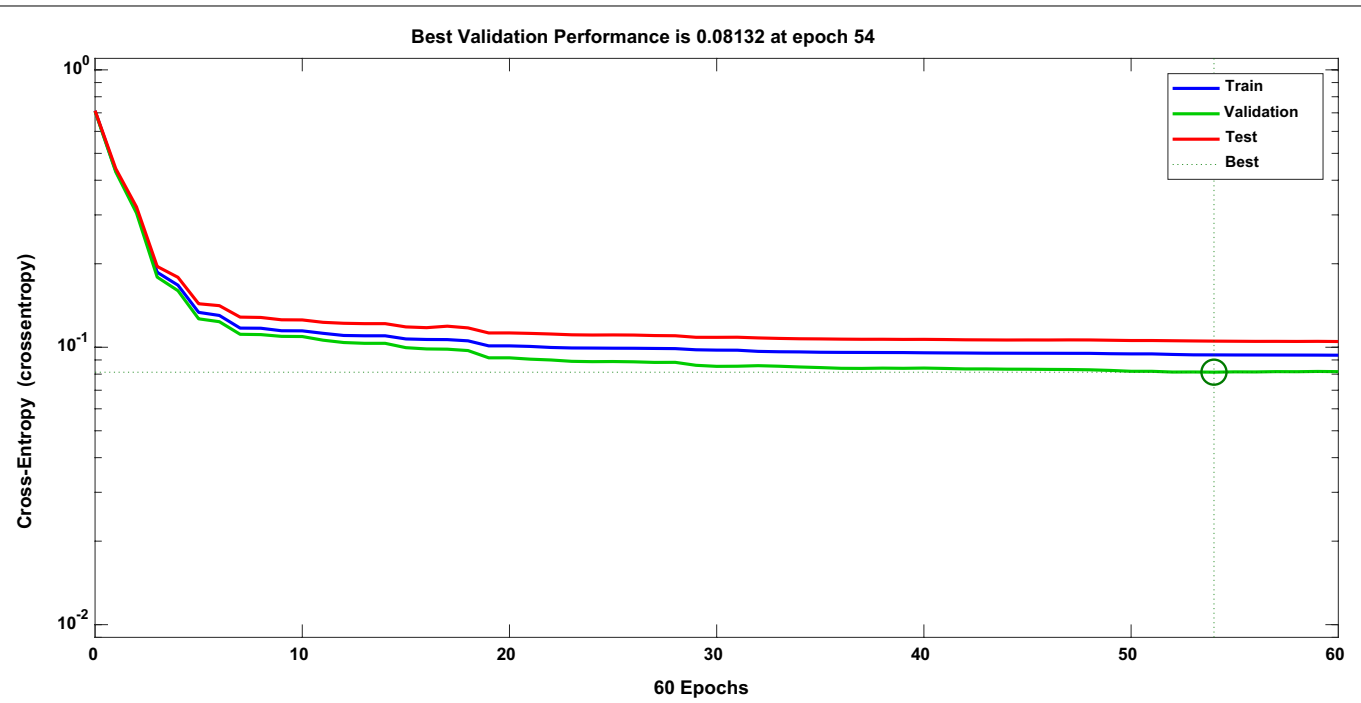

Fig. 4 Showing best validation performance at epoch 60

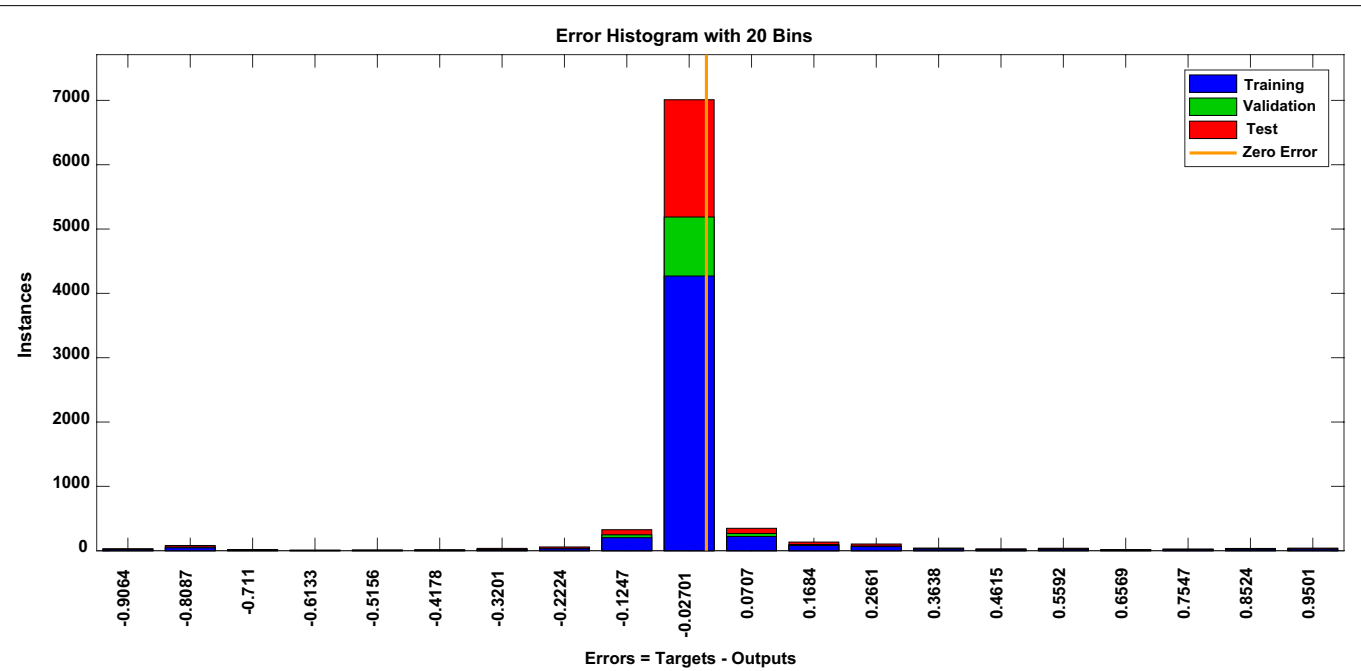

Fig. 5 Histogram of each testing validation and training state

and the testing state. As it can be seen, error difference between target and predicted values is minimum, exhibiting the good convergence.

A GUI was built using MATLAB for automated classification of epileptic seizures using the trained model developed. The name assigned to the GUI developed was 'Aepitect', which stands for automated epileptic seizure detection. The GUI was designed in such a way that it displays the $20 \mathrm{~s}$ of EEG every page. Features were extracted at a segmentation length of $0.5 \mathrm{~s}$, and the same were used to classify using the trained model. Figure 6 shows the screenshot of 'Aepitect', and it was cross-validated with the neurologist and found $98.5 \%$ matching.
The button 'Select File' allows the user to select the patient file, and the button 'Biomark' performs preprocessing, feature extraction, classification, and biomarking.

The performance of the proposed approach was compared with the other existing studies reported earlier. Table 5 shows the comparison results between different studies. As it is seen from Table 5 , most of the studies have used single-channel EEG data from the University of Bonn and achieved better results. One should take the attention while comparing the performance of different methods since different EEG databases were used in their respective studies. University of Bonn database was found to be clean EEG, and it works well for all the 


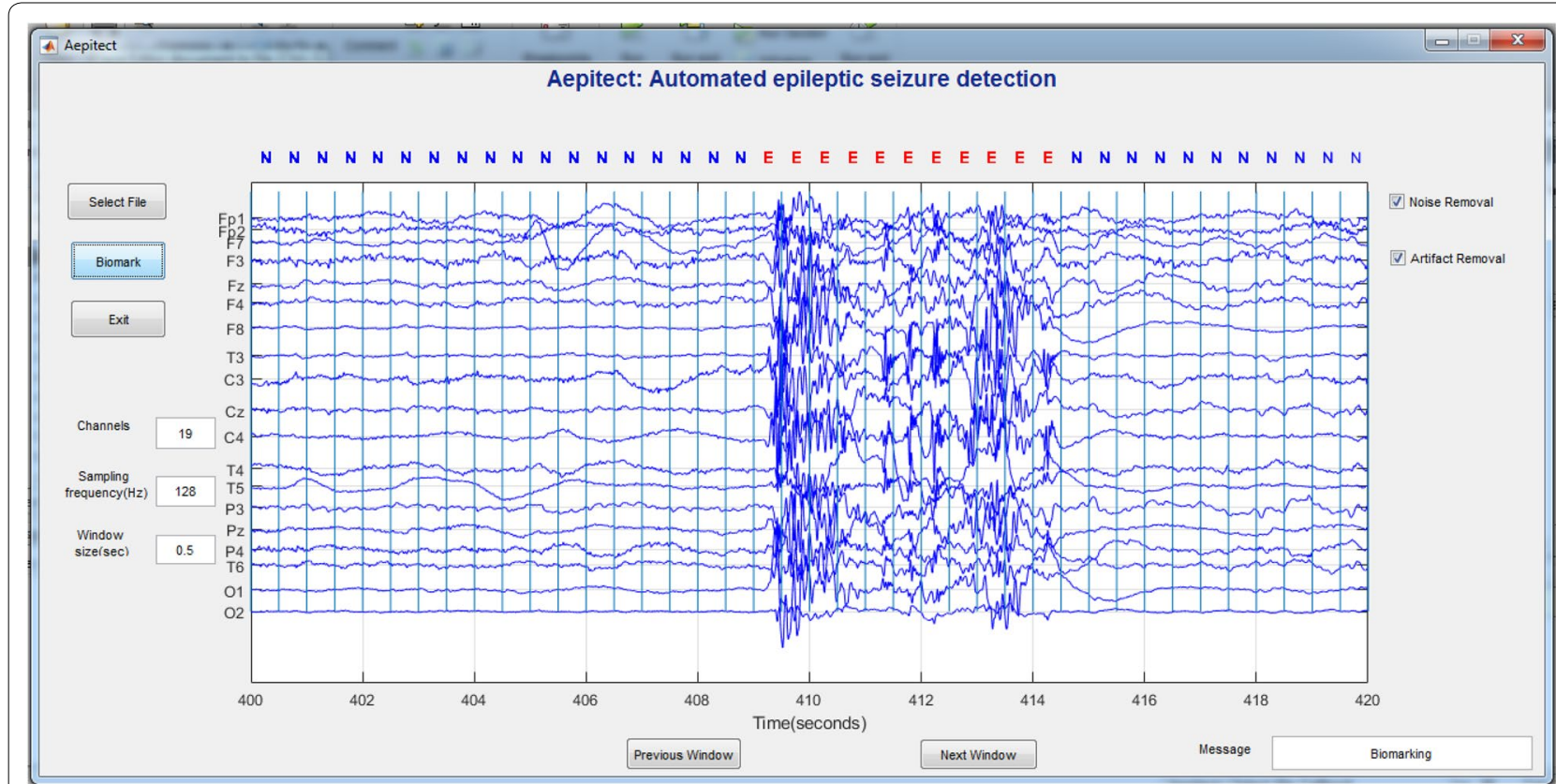

Fig. 6 Screenshot of GUI referred as 'Aepitect' developed in MATLAB

Table 5 Comparison results of some epileptic seizure detection methods

\begin{tabular}{|c|c|c|c|c|}
\hline Author & Features & Classifier & Results & Database \\
\hline Kiymik et al. & Autoregressive features & Back-propagation neural network & Accuracy 95\% & $\begin{array}{l}\text { Neurology department of the } \\
\text { Medical Faculty Hospital of } \\
\text { Dicle University }\end{array}$ \\
\hline Orhan et al. & DWT-based features & MLPNN & Accuracy 99.6 & University of Bonn \\
\hline Kamath 2013 & Teager energy & $\begin{array}{l}\text { Radial basis function neural } \\
\text { network }\end{array}$ & Accuracy $97.8 \%$ & University of Bonn \\
\hline Gurwinder et al. 2015 & $\begin{array}{l}\text { Wavelet transformation and spike- } \\
\text { based features }\end{array}$ & MLPNN & Accuracy 98.6 & University of Bonn \\
\hline Ahammad et al. & $\begin{array}{l}\text { Energy, entropy, standard devia- } \\
\text { tion, maximum, minimum, and } \\
\text { mean }\end{array}$ & MLPNN & Accuracy 84.2 & University of Bonn \\
\hline Wang et al. 2011 & Wavelet packet entropy & K-NN & Accuracy $100 \%$ & University of Bonn \\
\hline Abbasi et al. 2017 & DWT-based features & MLPNN & $98.33 \%$ & University of Bonn \\
\hline Srinivasan et al. 2007 & ApEn & Recurrent Elman neural network & Accuracy $100 \%$ & University of Bonn \\
\hline Proposed method & PSD, entropy, and Teager energy & MLPNN & $\begin{array}{l}\text { Sensitivity } 97.8 \% \\
\text { Specificity } 96.4 \% \\
\text { FDR } 1 \mathrm{~h}^{-1}\end{array}$ & $\begin{array}{l}\text { Ramaiah Memorial College and } \\
\text { Hospital, Bengaluru }\end{array}$ \\
\hline
\end{tabular}

methods. However, the challenge arises while dealing with long-term multi-channel EEG. Therefore, we have used our database for the study to overcome the existing issues such as less sensitivity, specificity, and FDR.

The results of seizure detection algorithms are usually evaluated based on the sensitivity of the raised alarms (number of detected seizures/total number of seizures) and false detection rate; it is not evaluated by the sensitivity and specificity of epochs/segments. It was noticed that studies using University of Bonn database had classified epileptic seizures as epochs/segments instead of detecting them as a complete seizure. When comparing with other methods, our method follows the evaluation criteria of sensitivity and FDR to evaluate the performance of the algorithm. As compared to other methods listed in Table 5, the proposed method matches the results of other studies without using any DWT on EEG signal. 
The significant contributions of the proposed study were:

1. EEG data were recorded at Ramaiah memorial hospital, Bengaluru, and were used for the study.

2. Artifacts were removed automatically using ICA technique and experts validated same at Ramaiah memorial hospital, Bengaluru.

3. From the preliminary study, the best PSD method (Yule-Walker) was selected for the feature extraction.

4. Three features, namely PSD, variants of entropy, and Teager energy, were used for the feature extraction.

5. The descriptive analysis shows the noticeable band difference between normal and epileptic EEG activities.

6. Wilcoxon rank-sum test shows the evidence to reject the null hypothesis at the $5 \%$ significance level.

7. Classification results show the better performance using multi-features as compared to the single features.

8. A MATLAB GUI called 'Aepitect' was developed for automated detection.

The above findings suggest that the proposed method is suitable for automated detection of epileptic seizures in real time. The complete study was implemented in MATLAB 2016b using 8 GB RAM, CPU $2 \mathrm{GHz}$ with Intel i5 processor. As a future step, more features will be included to increase the sensitivity and decrease the FDR. Further, deep learning concept will be explored for the classification of epileptic seizures.

\section{Conclusion}

This study provides a multi-channel EEG analysis for the detection of epileptic seizures using PSD, entropy, Teager energy, and MLPNN classifier. Initially, EEG signals were preprocessed to remove noise and artifacts, and features were extracted. Descriptive analysis and Wilcoxon rank-sum test proved the suitability of the extracted features for classification with noticeable band difference between normal and epileptic EEG. The simulation results showed sensitivity, specificity, and false detection rate of $97.8 \%, 96.4 \%$, and $1 \mathrm{~h}^{-1}$, respectively, using multi-features. Results indicate that the proposed study is suitable for real-time seizure recognition from multi-channel EEG recording. The graphical user interface referred as 'Aepitect' was developed in MATLAB to provide an automated biomarker for normal and epileptic EEG signals. It is anticipated that the proposed algorithm will offer a faster and accurate diagnosis and also reduce the time spent on detecting seizures from long-term multi-channel EEG recordings and can be extended to more patients for long-term EEG.

\section{Authors' contributions}

NS assisted on giving ideas and directions. SR and KT drafted and revised the manuscript together. KT, LN, and MK wrote MATLAB codes. ASH and ABK validation the clinical results. All authors read and approved the final manuscript.

\section{Author details}

${ }^{1}$ Centre for Medical Electronics and Computing, Ramaiah Institute of Technology (Affiliated to VTU Belgaum), Bengaluru, India. ${ }^{2}$ Institute of Neuroscience, Ramaiah Medical College and Hospitals, Bengaluru, India.

\section{Acknowledgements}

The authors would like to acknowledge the doctors of the Institute of Neurosciences, Ramaiah Memorial College and Hospital, Bengaluru, India, for permitting to use the EEG data for research.

\section{Authors' Information}

N. Sriraam received B.E. degree in Electronics and Communication Engineering in 1996, M.Tech. degree (with distinction) in Biomedical Engineering from MIT Manipal, India, in 2000, and Ph.D. degree in Information Technology from the Multimedia University, Cyberjaya, Malaysia, in the area of biomedical signal processing, in 2007. He is currently working as Professor and Head, Department of Medical Electronics, Ramaiah Institute of Technology, Bengaluru, India. $\mathrm{He}$ is the author or coauthor of 150 articles published in journals and has been involved in several sponsored research projects. His current research interests include biomedical signal processing, data mining and neural networks. He is a senior member of IEEE and IEEE Engineering in Medicine and Biology Society (EMBS), Signal Processing Society, life member of Telemedicine Society of India and member of Medical Computer Society of India.

S. Raghu received M.Tech. degree in Communication System from Christ University Faculty of Engineering in 2016 and M.Sc. degree from Bangalore University in 2011. He worked as Research Associate at Center for Medical Electronics and Computing, Ramaiah Institute of Technology, Bengaluru, India, from April 2016 to April 2017. Also, he worked as a lecturer at Bangalore City College from February 2012 to May 2014. His current research includes biomedical signal processing, machine learning and telemedicine.

Kadeeja Tamanna is a B.E. graduate in the field of Medical Electronics from Ramaiah Institute of Technology (2017). She is currently involved in the ongoing research project, and her study areas include biomedical signal processing and machine learning.

Leena Narayan is a B.E. graduate in the field of Medical Electronics from Ramaiah Institute of Technology (2017).

Mehraj Khanum is a B.E. graduate in the field of Medical Electronics from Ramaiah Institute of Technology (2017).

A. S. Hegde is a Neurosurgeon, Professor Head at Institute of Neurosciences, Ramaiah Medical College and Hospitals, Bengaluru, India. He is a member of Indian Society of Oncology, Indian Epilepsy Association, Neurological Society of India and World Federation of Neurological Surgeons. His research interests are genomics of cerebral glioma, medical economics, Ayurvedic medications in degenerative disorders of brain and telemedicine.

Anjani Bhushan Kumar is a Neurophysiology Technologist at Institute of Neurosciences, Ramaiah Medical College and Hospitals, Bengaluru, India.

\section{Competing interest}

The authors declare that they have no competing interests.

\section{Publisher's Note}

Springer Nature remains neutral with regard to jurisdictional claims in published maps and institutional affiliations.

Received: 22 May 2018 Accepted: 10 August 2018

Published online: 03 September 2018 


\section{References}

1. Dabye AS, Issaka M, Gueye L (2015) Localization of epileptic seizure with an approach based on the PSD with an auto-regressive model. arXiv :1506.00947

2. Kamath C (2013) A new approach to detect epileptic seizures in Electroencephalograms using Teager energy. ISRN Biomedical Engineering, Hindawi. https://doi.org/10.1155/2013/358108

3. Kamath C (2013) Teager energy based filter-Bank Cepstra in EEG classification for seizure detection using radial basis function neural network. ISRN Biomedical Engineering, Hindawi. https://doi.org/10.1155/2013/498754

4. Abbasi R, Esmaeilpour M (2017) Selecting statistical characteristics of brain signals to detect epileptic seizures using discrete wavelet transform and perceptron neural network. IJIMAI 4(5):33-38

5. Zhou W, Liu Y, Yuan Q, Li X (2013) Epileptic seizure detection using lacunarity and Bayesian linear discriminant analysis in intracranial EEG. IEEE Trans Biomed Eng 60(12):3375-3381

6. Ji Z, Sugi T, Goto S, Wang X, Ikeda A (2015) An automatic spike detection system based on elimination of false positives using the large-area context in the scalp EEG. IEEE Trans Biomed Eng 58:2478-2488

7. Xia YS, Leung H (2006) Nonlinear spatial-temporal prediction based on optimal fusion. IEEE Trans Neural Netw 17:975-988

8. Gotman J (1982) Automatic recognition of epileptic seizures in the EEG. Electroencephalogr Clin Neurophysiol 99:530-540

9. Guo L, Rivero D, Pazos A (2010) Epileptic seizure detection using multiwavelet transform based approximate entropy and artificial neural networks. J Neurosci Methods 193:156-163. https://doi.org/10.1016/j. jneumeth.2010.08.030

10. Kiymik MK, Subasi A, Ozcalık HR (2004) Neural networks with periodogram and autoregressive spectral analysis methods in detection of epileptic seizure. J Med Syst 28(6):511-522

11. Orhan U, Orhan M, Ozer M (2011) EEG signals classification using the K-means clustering and a multilayer perceptron neural network model. Expert Syst Appl 38(10):13475-13481

12. Gurwinder S, Kaur M, Dalwinder S (2015) Detection of epileptic seizure using wavelet transformation and spike-based features. In: 2nd international conference on recent advances in engineering \& computational sciences (RAECS) 2015, pp 1-4

13. Ahammad N, Fathima T, Joseph P (2014) Detection of epileptic seizure event and onset using EEG. Biomed Res Int 2014:450573

14. Wang D, Miao D, Xie C (2011) Best basis-based wavelet packet entropy feature extraction and hierarchical EEG classification for epileptic detection. Expert Syst Appl 38(11):14314-14320

15. Menshawy ML, Benharref A, Serhani M (2015) An automatic mobilehealth based approach for EEG epileptic seizures detection. Expert Syst Appl 42:7157-7174

16. Bogaarts JG et al (2016) Optimal training dataset composition for SVM based age independent, automated epileptic seizures detection. J Med Bio-logical Eng Comput 54:1285-1293

17. Das AB, Bhuiyan MIH (2016) Discrimination and classification of focal and non-focal EEG signals using entropy-based features in the EMD-DWT domain. Biomed Signal Process Control 29:11-21
18. Raghu S, Sriraam N (2018) Classification of focal and non-focal EEG signals using neighborhood component analysis and machine learning algorithms. Expert Syst Appl 113:18-32

19. Srinivasan V, Eswaran C, Sriraam N (2007) Artificial neural network based epileptic detection using time-domain and frequency-domain features. $J$ Med Syst 29(6):647-660

20. Raghu S, Sriraam N, Pradeep KG (2015) Effect of wavelet packet log energy entropy on electroencephalogram (EEG) signals. Int J Biomed Clin Eng 4(1):32-43

21. Raghu S, Sriraam N, Pradeep KG (2017) Classification of epileptic seizures using wavelet packet log energy and norm entropies with recurrent Elman neural network classifier. Cogn Neurodyn 11(1):51-66

22. Pravin SK, Sriraam N, Benakop PG, Jinaga BC (2010) Entropies based detection of epileptic seizures with artificial neural network classifiers. Expert Syst Appl 37:3284-3291

23. Acharya UR, Molinari F, Vinitha SS, Chattopadhyay S, Kwan-Hoong N, Suri JS (2012) Automated diagnosis of epileptic EEG using entropies. Biomed Signal Process Control 7(4):401-408

24. Adeli H, Zhou Z, Dadmehr N (2003) Analysis of EEG records in an epileptic patient using wavelet transform. J Neurosci Methods 123(1):69-87

25. Srinivasan V, Eswaran C, Sriraam N (2007) Approximate entropy-based epileptic EEG detection using artificial neural networks. IEEE Trans Inf Technol Biomed 11(3):288-295. https://doi.org/10.1109/TITB.2006.884369

26. Sriraam N, Raghu S (2017) Classification of focal and non focal epileptic seizures using multi-features and SVM classifier. J Med Syst 41(10):160

27. Raghu S, Sriraam N (2017) Optimal configuration of multilayer perceptron neural network classifier for recognition of intracranial epileptic seizures. Expert Syst Appl 89:205-221

28. Cardoso JF (1998) Blind signal separation: statistical principles. Proc IEEE 86(10):2009-2025

29. Cichocki A, Vorobyov S (2000) Application of ICA for automatic noise and interference cancellation in multisensory biomedical signals. In: Proceedings of the 2nd internationl workshop on ICA and BSS 2000, pp 621-626

30. Uriguen JA, Zapirain BG (2015) EEG artifact removal-state-of-the-art and guidelines. J Neural Eng 12(3):031001

31. Stoica P, Moses RL (2005) Introduction to spectral analysis. Prentice Hall, Upper Saddle River, p 07458

32. Shannon CE (1948) A mathematical theory of communication. Bell Syst Tech J 27:379-423

33. Renyi A (1961) On measures of information and entropy. In: Proceedings of the fourth Berkeley symposium on mathematics, statistics and probability, pp 547-561

34. Teager HM, Teager SM (1983) A Phenomenological Model for Vowel Production in the Vocal Tract, vol 3. College-Hill Press, San Diego, pp 73-109

35. Probability and Statistics (2014) http://www.statisticshowto.com/proba bility-and-statistics/z-score. Accessed 20 Jan 2018

36. Elman JL (1990) Finding structure in time. Cogn Sci 14:179-211

37. Sriraam N (2012) EEG based automated detection of auditory loss: a pilot study. Expert Syst Appl 39(1):723-731

38. Tang Z, Li R (2001) An improved neural network model and its applications. J Inf Comput Sci 8(10):881-1888

\section{Submit your manuscript to a SpringerOpen ${ }^{\circ}$ journal and benefit from:}

- Convenient online submission

- Rigorous peer review

- Open access: articles freely available online

- High visibility within the field

Retaining the copyright to your article

Submit your next manuscript at $>$ springeropen.com 